\title{
Estudio comparativo de la dosis de diálisis medida por dialisancia iónica (Kt) y por Kt/V
}

\author{
Premio de Investigación en Enfermería Nefrológica Janssen- Cilag
}

\section{Ana Vanessa Fernández Martínez* - Salvadora Soto Ureña* - María Arenas Fuentes* - Natalia Sáez Donaire* - $\mathbf{M}^{\mathrm{a}}$ Mercedes Gracia Canovas** - Patricia Ortega Hernández**}

*Unidad de Cartagena - **Unidad de San Pedro del Pinatar - Centros de Hemodiálisis Nefroclub Carthago

\section{Resumen}

La dosis de diálisis administrada influye en la supervivencia de los pacientes, siendo deseable alcanzar un Kt/V óptimo. Diferentes estudios demuestran que existe una buena correlación entre el Kt medido por dialisancia (sin necesidad de extracciones sanguíneas) y el Kt/V.

El objetivo de este estudio prospectivo fue comparar la medición de la dosis de diálisis por ambos métodos (Kt y Kt/V) en nuestra población en hemodiálisis, así como aquellos factores relacionados con la técnica que influyen en la dosis de diálisis alcanzada.

Se ha estudiado a 131 pacientes. No se aprecian diferencias significativas en el Kt en función del sexo mientras que el $\mathrm{Kt} / \mathrm{V}$ se muestra como significativamente mayor en las mujeres $(1,93 \pm 0,23)$ que en los hombres $(1,59 \pm 0,25)$.

Mientras que en el Kt las diferencias son significativas en función del acceso vascular no lo son para el Kt/V. En la dosis de diálisis en función del tipo de hemodiálisis, no se aprecian diferencias signifi-

Correspondencia:

Ana Vanessa Fernández Martínez

Nefroclub Carthago. Fresenius Medical Care

Budapest, parcela 136

Polígono Industrial Cabezo Beaza

30353 Cartagena. Murcia

nefroclubcarthago@gmail.com cativas en $\mathrm{Kt} / \mathrm{V}$ entre los que reciben tratamiento con HD convencional y los que lo hacen con hemodiafiltración on-line, mientras que el $90 \%$ de los pacientes en hemodiafiltración on-line alcanzan un Kt óptimo frente a un 45,5\% en HD convencional. La monitorización continua de la dosis de diálisis mediante la medición del Kt por dialisancia iónica permite discriminar mejor a los pacientes en situación de infradiálisis que la determinación del Kt/V.

\section{PALABRAS CLAVE: DOSIS DE DIÁLISIS \\ - KT \\ - KT/V \\ - HEMODIÁLISIS \\ - HEMODIAFILTRACIÓN}

Comparative study of the dialysis dose measured by ionic dialysance (Kt) and by Kt/V

\section{Abstract}

The dialysis dose administered affects patient survival, and it is desirable to attain an optimal Kt/v. Different studies show that there is a good correlation between the Kt measured by dialysance (with no need for blood extractions) and the Kt/V.

The aim of this prospective study was to compare the measurement of the dialysis doses using both methods ( $\mathrm{Kt}$ and $\mathrm{Kt} / \mathrm{V}$ ) in our haemodialysis population, as well as factors related to the technique that affect the dialysis dose reached. 
131 patients were studied. No significant differences were observed in $\mathrm{Kt}$ depending on sex while $\mathrm{Kt} / \mathrm{V}$ is significantly higher in women $(1.93 \pm 0.23)$ than in men $(1.59 \pm 0.25)$.

While in Kt the differences are significant depending on the vascular access, they are not for Kt/V. In the dialysis dose depending on the type of haemodialysis, no significant differences were observed in $\mathrm{Kt} / \mathrm{V}$ between those who receive treatment with conventional $\mathrm{HD}$ and those undergoing on-line haemodiafiltration, while $90 \%$ of the patients undergoing on-line haemodiafiltration reach an optimal $\mathrm{Kt}$ compared to $45.5 \%$ in conventional HD. Continuous monitoring of the dialysis dose by measuring the Kt through ionic dialysance makes it possible to discriminate better which patients are in a situation of infradialysis than by determining $\mathrm{Kt} / \mathrm{V}$.

\section{KEY WORDS}
- DIALYSIS DOSE
- KT
$-\mathrm{KT} / \mathrm{V}$
- HAEMODIALYSIS
- HAEMODIAFILTRATION

\section{Introducción}

La dosis administrada de diálisis influye en la supervivencia del paciente en hemodiálisis ${ }^{1}$, siendo la recomendación más generalizada alcanzar un valor de $\mathrm{Kt} / \mathrm{V}$ mayor de 1,3, pudiendo beneficiarse de valores superiores $(1,6)$ las mujeres en hemodiálisis $^{2-3}$. Por otro lado, la determinación del Kt/V exige la extracción de muestras de urea sanguínea en unas condiciones precisas, pudiendo alterar sus resultados la inadecuación del protocolo.

Mediante dos medidas de la conductividad del líquido de diálisis a la entrada y a la salida del dializador se aplica un modelo matemático, de dos ecuaciones para dos incógnitas, que nos permite conocer la dialisancia iónica efectiva -que es el valor de dialisancia de electrolitos corregidos para la ultrafiltración y la recirculación del acceso vascular- y la conductividad del agua plasmática. La dialisancia iónica efectiva es equivalente al $\mathrm{K}$ de urea. La medición sistemática del $\mathrm{K}$ por el tiempo transcurrido de diálisis permite obtener el Kt, una forma real de medir la dosis de diálisis, expresada en litros. Trabajar con el Kt tiene ventajas, tanto el $\mathrm{K}$ como el $\mathrm{t}$ son reales y medidos por el monitor. Si pautamos el Kt/V debemos introducir el $\mathrm{V}$ que puede ser erróneo y manipulable durante la sesión.

Diferentes estudios ${ }^{4}$ que han utilizado la dialisancia iónica en hemodiálisis lo expresan como Kt/V y llegan a la conclusión de que el Kt/V medido por dialisancia iónica es diferente al medido por analítica, aunque existe una buena correlación demostrando que existe una variabilidad intermétodo. El $\mathrm{Kt} / \mathrm{V}$ determinado con dialisancia iónica normalmente infraestima cuando se compara con el $\mathrm{Kt} / \mathrm{V}$ calculado con la fórmula de Daugirdas de segunda generación obtenida con la analítica.

Desde 1999 Lowrie y col ${ }^{5}$ proponen el Kt como marcador de dosis de diálisis y mortalidad recomendando un Kt mínimo de 40-45 litros para las mujeres y 45-50 para los hombres. En un estudio de 3009 pacientes ${ }^{6}$, observaron una curva de supervivencia en $\mathrm{J}$ cuando distribuyeron los pacientes en quintiles según el PRU mientras que la curva era descendente cuando se utilizaba el Kt, es decir que un mayor Kt se acompañaba de una mayor supervivencia. Recientemente Maduell y col$^{7}$ identifican el Kt como mejor indicador de dosis de diálisis en situación de infradiálisis.

Desde el punto de vista de la actuación enfermera, la medición continua de la dosis de diálisis mediante el Kt permite conocer la eficacia dialítica en cada sesión de diálisis, sin que para ello sea preciso realizar extracciones sanguíneas seriadas. Del mismo modo, permite alertar sobre problemas que puedan influir negativamente sobre el desarrollo de la sesión, y por tanto establecer las medidas de corrección oportunas en la misma sesión de diálisis.

El objetivo de este estudio prospectivo fue comparar la medición de la dosis de diálisis por ambos métodos ( Kt y Kt/V) en nuestra población en hemodiálisis, así como aquellos factores relacionados con la técnica que influyen en la dosis de diálisis alcanzada. 


\section{Material y Métodos}

Se trata de un estudio prospectivo sobre pacientes con enfermedad renal crónica en tratamiento sustitutivo con hemodiálisis de nuestra área de salud, mayores de 18 años que sean tratados con monitores de diálisis que permitan la medición del Kt por dialisancia iónica.

En todos los pacientes incluidos se procede a la medición de Kt mediante dialisancia iónica OCM Fresenius Medical Care ${ }^{\circledR}$ durante tres sesiones consecutivas, así como a la determinación del Kt/V (Dauguirdas $2^{\mathrm{a}}$ generación). Para la obtención de la muestra de urea postdiálisis según protocolo de nuestra unidad, a la finalización de la sesión y con la ultrafiltración total alcanzada, se reduce el flujo sanguíneo a $100 \mathrm{ml} / \mathrm{mín}$. , y tras 15 segundos de espera, se para la bomba arterial y se extrae la muestra sanguínea del botón arterial del circuito extracorpóreo.

Variables en estudio: Kt (promedio de tres sesiones consecutivas de hemodiálisis) y $\mathrm{Kt} / \mathrm{V}$, variables demográficas (edad, sexo, tiempo de insuficiencia renal terminal (IRT), etiología de IRT) y otras variables relacionadas con la diálisis (acceso vascular, tipo de hemodiálisis, dializador, peso seco, tiempo prescrito y flujo efectivo).

Se establecen tres grupos en función de los resultados de Kt. Grupol: Kt óptimo ( Kt superior a 50 litros en hombres y 45 litros en mujeres). Grupo 2: Kt normal (Kt entre 45 y 50 litros en hombres y 40 a 45 litros en mujeres) y Grupo 3: Kt bajo (Kt menor de 45 litros en hombres y 40 litros en mujeres). $Y$ otros 2 grupos en función del Kt/V alcanzado: $\mathrm{Kt} / \mathrm{V}$ mayor o igual a 1,3 y $\mathrm{Kt} / \mathrm{V}$ menor de 1,3 .

Los parámetros del estudio se recogen en la historia clínica y en las gráficas de enfermería. En cada sesión se anota el Kt alcanzado, y en el caso de superar los límites descritos para Kt óptimo, se indica el momento de la sesión de hemodiálisis cuando se produce.

El análisis estadístico se realiza con el programa estadístico SPSS 13.0 para Windows. Las variables cuantitativas se expresan como media, desviación estándar y rango. Las variables cualitativas, como frecuencia y porcentaje. El contraste de hipótesis para variables cuantitativas se realiza mediante la t-student y el análisis de la varianza (ANOVA), y la chicuadrado de Pearson para variables cualitativas.

\section{Resultados}

Se estudió a 131 pacientes; sus características basales se describen en la tabla 1 . No se aprecian diferencias significativas en el Kt en función del sexo (49,18 $\pm 8,13$ litros en hombres frente a 47,73 $\pm 5,30$ litros

Tabla 1: Características basales.

\begin{tabular}{|c|c|}
\hline Parámetro & Valor \\
\hline Edad, años & $63,4 \pm 15,4$ (rango 19-89) \\
\hline $\begin{array}{l}\text { Sexo, n }(\%) \\
\text { Hombres } \\
\text { Mujeres } 37 \\
\end{array}$ & $\begin{array}{l}93(71) \\
38(29)\end{array}$ \\
\hline Permanencia en IR terminal, meses & $56,9 \pm 65,9$ (rango 3-306) \\
\hline $\begin{array}{c}\text { Causa de insuficiencia renal, } \mathrm{n}(\%) \\
\text { Desconocida } \\
\text { Glomerular } \\
\text { Vascular } \\
\text { Diabetes } \\
\text { Hereditária } \\
\text { Intersticial } \\
\text { Otras causas }\end{array}$ & $\begin{array}{l}27(20,6) \\
19(14,5) \\
26(19,8) \\
20(15,3) \\
22(16,8) \\
15(11,5) \\
2(1,5)\end{array}$ \\
\hline $\begin{array}{l}\text { Tipo de acceso vascular, n (\%) } \\
\text { FAV nativa } \\
\text { FAV protésica } \\
\text { CPT }\end{array}$ & $\begin{array}{l}112(85,5) \\
8(6,1) \\
11(8,4) \\
\end{array}$ \\
\hline Tiempo sesión HD, minutos & $238,82 \pm 7,17$ (rango 195-260) \\
\hline $\begin{array}{r}\text { Dializador, } \mathrm{n}(\%) \\
\text { Helixona } 1.3 \mathrm{~m} 2 \\
\text { Helixona 1,6 m2 }\end{array}$ & $\begin{array}{l}114(87) \\
17(13 \%)\end{array}$ \\
\hline $\begin{array}{l}\text { Técnica de diálisis,n (\%) } \\
\text { Convencional } \\
\text { Hemodiafiltración en línea }\end{array}$ & $\begin{array}{l}101(77,1) \\
30(22,9)\end{array}$ \\
\hline Peso seco, Kg. & $60,1 \pm 13,69$ \\
\hline Kt, litros & $48,76 \pm 7,43$ (rango $24,4-66,3)$ \\
\hline $\mathrm{Kt} / \mathrm{V}$ & $1,69 \pm 0,29($ rango $1-2,35)$ \\
\hline
\end{tabular}


en mujeres). Sin embargo, el Kt/ $\mathrm{V}$ se muestra como significativamente $(p<0,001)$ mayor en las mujeres $(1,93 \pm 0,23)$ que en los hombres $(1,59 \pm 0,25)$.

En cuanto a la distribución de grupos por la medición de Kt en función del sexo y Kt/V, los resultados se muestran en la figura 1 . Se aprecian diferencias significativas en el porcentaje de pacientes infradializados en función del método de determinación ( $p=$ 0,008 ). Así, mientras que solo el $7,3 \%$ de nuestra muestra presenta un $\mathrm{Kt} / \mathrm{V}<1,3$, el $16 \%$ no alcanzaría el Kt normal en función del sexo. El 55,7\% de los pacientes alcanzan un Kt óptimo, siendo el porcentaje significativamente $(p=0,010)$ mayor en la mujer $(76,3 \%)$ que en el hombre $(47,3 \%)$. Los resultados por sexos se aprecian en la figura 2.

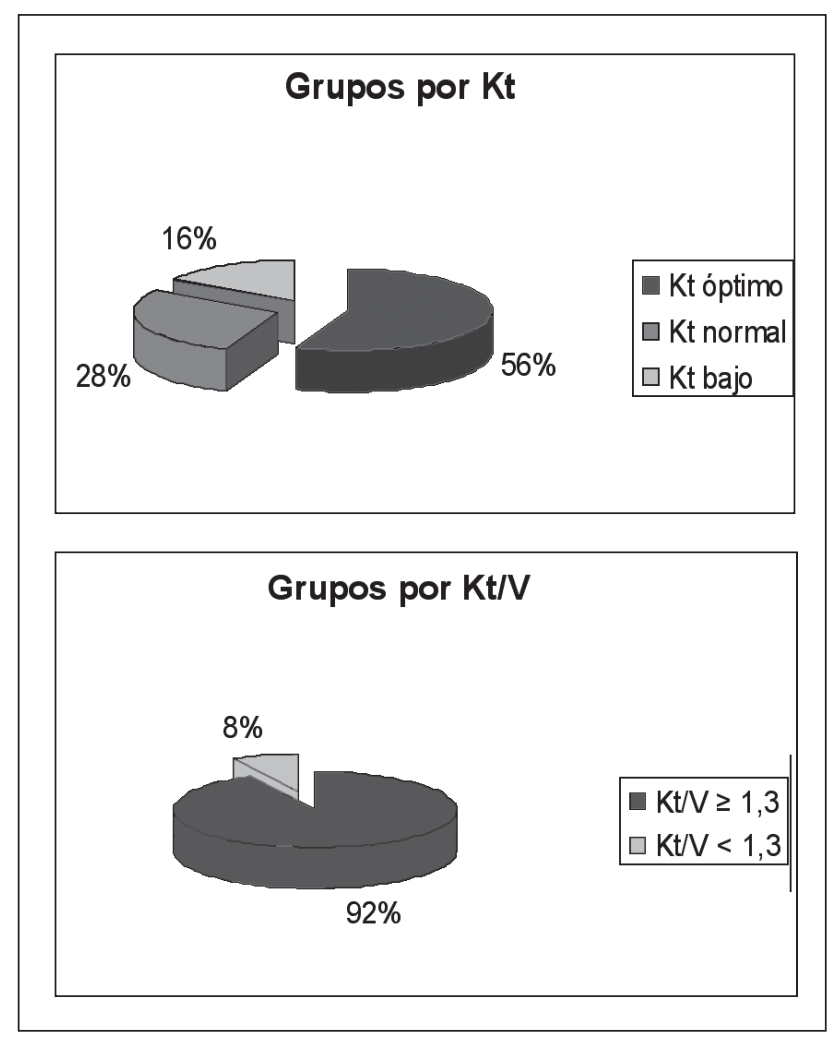

Figura 1. Grupos de pacientes en función de los resultados de $\mathrm{Kt}$ y $\mathrm{Kt} / \mathrm{V}$.

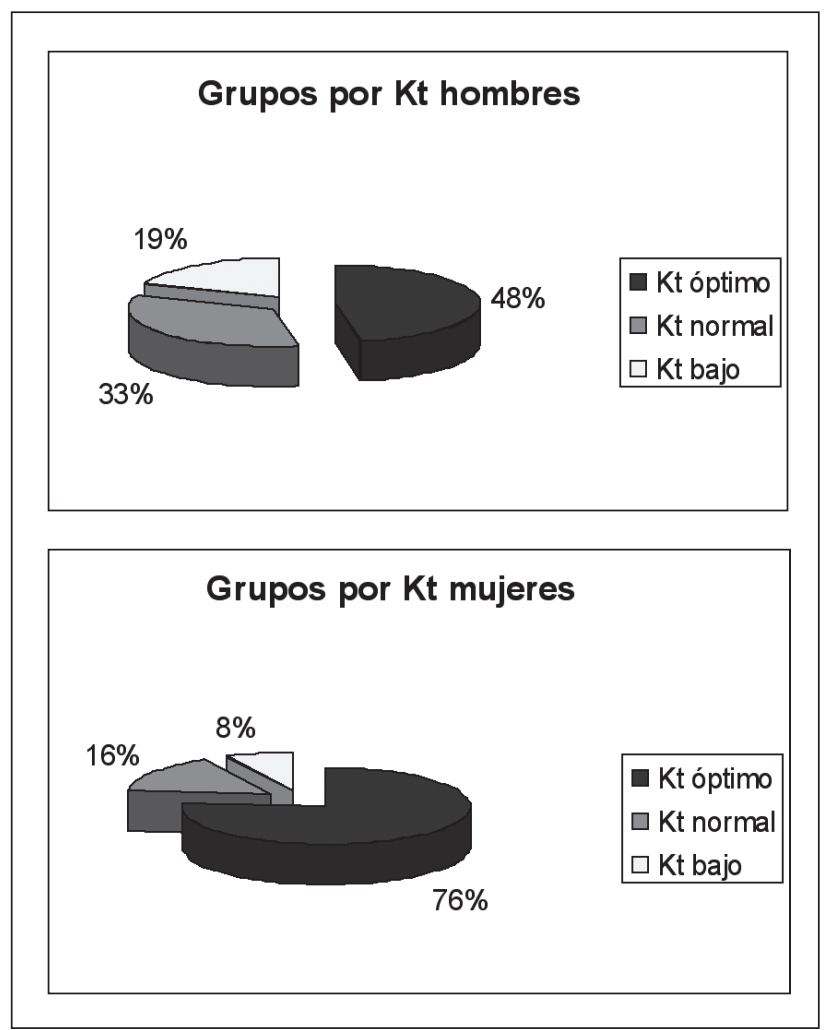

Figura 2. Distribución del Kt por sexos

Los datos de $\mathrm{Kt}$ y Kt/V según acceso vascular se muestran en la tabla 2. Mientras que en el Kt las diferencias son estadísticamente significativas ( $p=$ 0,008 ), no lo son para el $\mathrm{Kt} / \mathrm{V}$. En el paciente con fístula nativa se alcanzan 7,14 litros más de media que en el paciente con catéter $(p=0,002)$. El flujo sanguíneo efectivo es significativamente $(p<0,001)$ mayor en los pacientes que alcanzan el Kt óptimo que en los que alcanzan un Kt normal $(p=0,005)$ o bajo $(p<0,001)$. Los datos se reflejan en la figura 3 .

En los pacientes en los que se alcanza el Kt óptimo el tiempo trascurrido medido con respecto al prescrito en la sesión fue de $-20,7 \pm 13,9$ minutos (rango 1-54). Entre los que no lo alcanzan sería necesario un incremento estimado del tiempo por sesión de $36,91 \pm 43,41$ minutos (rango 1-214). El 63,3\% de

Tabla 2. Distribución de dosis de diálisis por acceso vascular.

\begin{tabular}{|l|l|l|l|l|}
\hline & FAV nativas & FAV protésicas & CPT & p (ANOVA) \\
\hline Kt, litros & $49,48 \pm 7,08$ & $47,63 \pm 6,12$ & $42,34 \pm 9,11$ & 0,008 \\
\hline Kt/V & $1,70 \pm 0,28$ & $1,79 \pm 0,18$ & $1,54 \pm 0,41$ & Ns \\
\hline
\end{tabular}


los pacientes que no alcanzan un Kt óptimo lo haría con incrementar el tiempo de la sesión 30 minutos o menos.

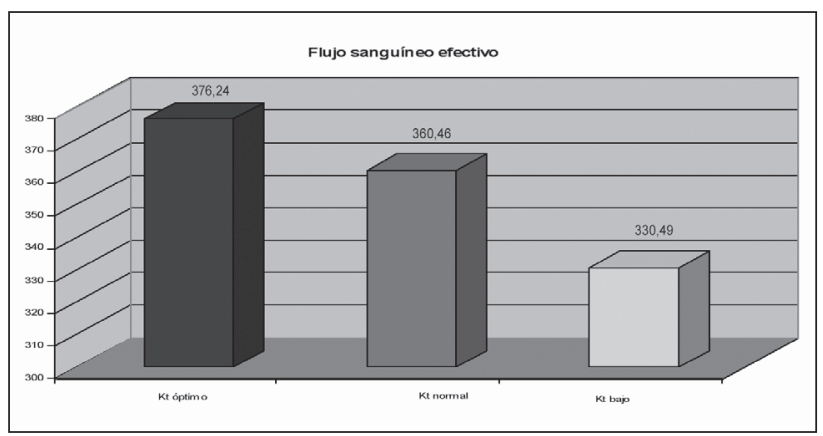

Gráfico 3: Flujo sanguíneo efectivo según Kt

Cuando se analiza la dosis de diálisis en función del tipo de hemodiálisis prescrito, no se aprecian diferencias significativas en $\mathrm{Kt} / \mathrm{V}$ entre los que reciben tratamiento con HD convencional $(1,67 \pm 0,28)$ y los que lo hacen con hemodiafiltración on-line $(1,74 \pm$ $0,32)$. Sin embargo, en estos últimos se observa un $\mathrm{Kt}$ significativamente $(p<0,001)$ mayor $(55,59 \pm 6,59$ frente a $46,74 \pm 6,40$ litros). El $90 \%$ de los pacientes en hemodiafiltración on-line alcanzan un Kt óptimo ( $87 \%$ en hombres y $100 \%$ en las mujeres) frente a un $45,5 \%$ en HD convencional $(34,3 \%$ en hombres y $71 \%$ en mujeres), diferencia estadísticamente significativa $(p=0,002)$. Los resultados se expresan en la figura 4.

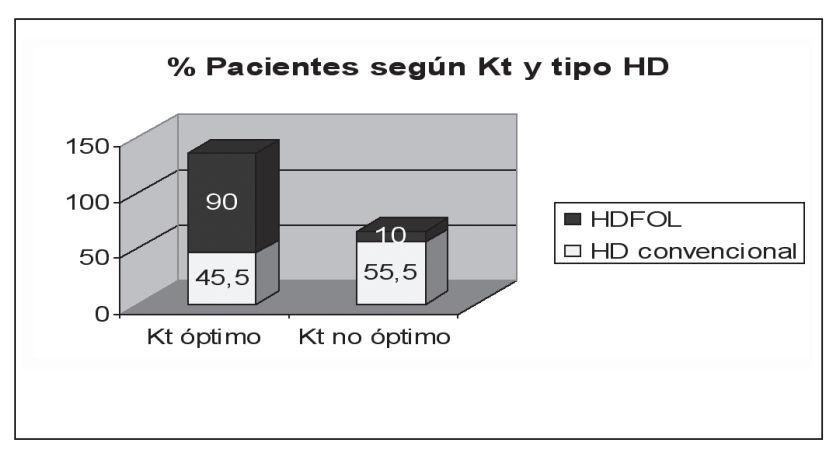

Gráfico 4: Distribución por Kt y tipo de hemodiálisis.

\section{Discusión}

La dosis de diálisis influye significativamente en la anemia, estado nutricional y, lo que es más importante, en la supervivencia de los pacientes en hemodiálisis ${ }^{1}$. Es por ello, que las diferentes guías de ámbito internacional incluyen recomendaciones de dosis de hemodiálisis mediante un $\mathrm{Kt} / \mathrm{V}$ mayor de $1,3^{2-3}$. Pero bien es sabido, que son numerosos factores los que condicionan el cumplimiento de la dosis prescrita en cada sesión de diálisis, por lo que resulta lógico que aparezcan nuevos métodos de medición en cada sesión de diálisis. Entre ellos, se encuentra el cálculo del Kt por dialisancia iónica, descrito hace casi una década ${ }^{5}$. Este parámetro obvia la $\mathrm{V}$ del $\mathrm{Kt} / \mathrm{V}$, que presenta una alta variabilidad y confusión en su estimación. El cálculo del Kt es reconocido por las Guías de la Sociedad Española de Nefrología ${ }^{8}$.

La medición del Kt ofrece ventajas, como la posibilidad de realizar una estimación contínua en cada sesión de la dosis de diálisis, y no siendo manipulable en el transcurso de la sesión ${ }^{7}$, y además no requiere de extracciones sanguíneas como en el caso del Kt/V medido con analítica, en el que además la determinación de la urea postdiálisis de forma inadecuada puede alterar notablemente los resultados obtenidos. Tal como se mencionó antes el Kt ha sido relacionado con la supervivencia del paciente por algunos autores ${ }^{6}$.

Así, muy recientemente Maduell y $\operatorname{col}^{7}$ describen un $31 \%$ de los pacientes que no alcanzan la dosis prescrita si tomamos como referencia el límite superior, y por tanto más exigente del Kt óptimo para cada sexo ( $34 \%$ en los hombres y $26 \%$ en las mujeres), pese a que toda su muestra alcanzaba un $\mathrm{Kt} / \mathrm{V}$ superior a 1,3 , lo que permite deducir que el Kt discrimina mejor las situaciones de infradiálisis, y además en cada sesión. Esto concuerda con nuestros resultados, ya que frente a un $7,6 \%$ de los pacientes con un $\mathrm{Kt} / \mathrm{V}$ menor de 1,3, un $44,3 \%$ de los mismos presentan un Kt no óptimo por sexo.

Por último, el Kt/V se ve influido por la desnutrición, patología frecuente en nuestros pacientes en hemodiálisis ${ }^{9}$, cuestión que no afecta al Kt.

Nuestro estudio relaciona la dosis de diálisis con otros factores bien conocidos en la prescripción de la diálisis adecuada ${ }^{10}$, como son el tiempo prescrito, el tipo de acceso vascular, el flujo sanguíneo efectivo y el tipo de hemodiálisis. Así, con la combinación de un acceso vascular (fístula nativa) adecuado, un incremento moderado en el tiempo de diálisis ( 30 minutos o menos), un flujo sanguíneo efectivo alto (> $370 \mathrm{ml} /$ min) y la prescripción de hemodiafiltración en línea, conseguiríamos en la inmensa mayoría de nuestros 
pacientes una dosis de diálisis tan exigente como la que proponen Maduell y col.

La implicación del personal de enfermería en la adecuación de estos parámetros es una pieza angular en el tratamiento de la hemodiálisis, tal como demuestra la relevancia de pequeños incrementos de flujos sanguíneos en la consecución del Kt prescrito ( $16 \mathrm{ml} /$ min). Se debe personalizar el tratamiento dialítico en cada una de sus facetas, para lo que la intervención enfermera es fundamental.

\section{Conclusiones}

La monitorización continua de la dosis de diálisis mediante la medición del Kt por dialisancia iónica permite discriminar mejor a los pacientes en situación de infradiálisis que la determinación del Kt/V. Por las características de esta medición, no hace precisa la extracción sanguínea seriada, reduciendo costes y cargas de enfermería.

Algunos factores como el tipo de acceso vascular del paciente, tipo de hemodiálisis, tiempo de hemodiálisis y flujo sanguíneo efectivo condicionan la dosis alcanzada de Kt en la sesión de hemodiálisis. Respetando los protocolos establecidos en cada unidad de hemodiálisis de seguridad del acceso vascular, se precisa un esfuerzo de individualización de cada paciente por parte del personal de enfermería para alcanzar el flujo efectivo óptimo de cada acceso vascular.

\section{Agradecimientos}

A todos los trabajadores de Nefroclub por su colaboración, al Dr. Maduell por habernos clarificado algunos conceptos y al Dr. Molina por su ayuda y apoyo incondicional.

\section{Bibliografía}

1. Help PJ, Port FK, Wolfe RA y col. The dose of hemodialysis and patients mortality. Kidney Int 1996; 50: 550-556.

2. NKF-DOQI Hemodialysis Adequacy Work Group Memberchip. Guidelines for hemodialysis adequacy. Am J Kidney Dis 1997; 30 (Suppl 2): S22S63.

3. European Best Practice Guidelines for Haemodialysis. Nephrol Dial Transplant 2002; 17 (Supplement 7):17-21.

4. Teruel JL, Fernández Lucas L, Marcel R y col. Cálculo de la dosis de diálisis mediante dialisancia iónica. Nefrología 2001; 21: 78-83.

5. Lowrie EG, Chertow GN, Lew NM y col. The urea (clearance $x$ dialysis time) product $(\mathrm{Kt})$ as an outcome-based measure of hemodialysis dose. Kidney Int 1999; 56: 729-737.

6. Chertow GM, Owen WF, Lazarus JM y col. Exploring the reverse j-saped curve between urea production ratio and mortality. Kidney Int 1999; 56:1872-1878.

7. Maduell F, Vera M, Serra N y cols. Kt como control y seguimiento de la dosis em uma unidad de hemodiálisis. Nefrología 2008; 28: 43-47.

8. Maduell F, García M, Alcazar R. Dosificación y adecuación del tratamiento dialítico. Guías SEN: Guías de centros de hemodiálisis. Nefrología 2006; 26 (Sup 8): 15-21.

9. Teruel JL, Fernández M. Dosis de diálisis. Dificultad de su medida. Nefrología 2008; 28(1):28-29.

10. Maduell F. Dosis de hemodiálisis: condición sine quanon de diálisis adecuada. Nefrología 1999.19; (Sup 4): 51-53. 